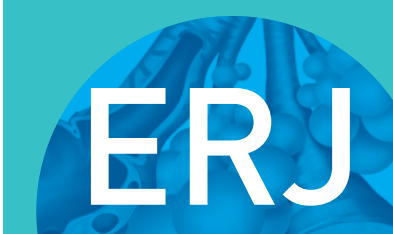

open research
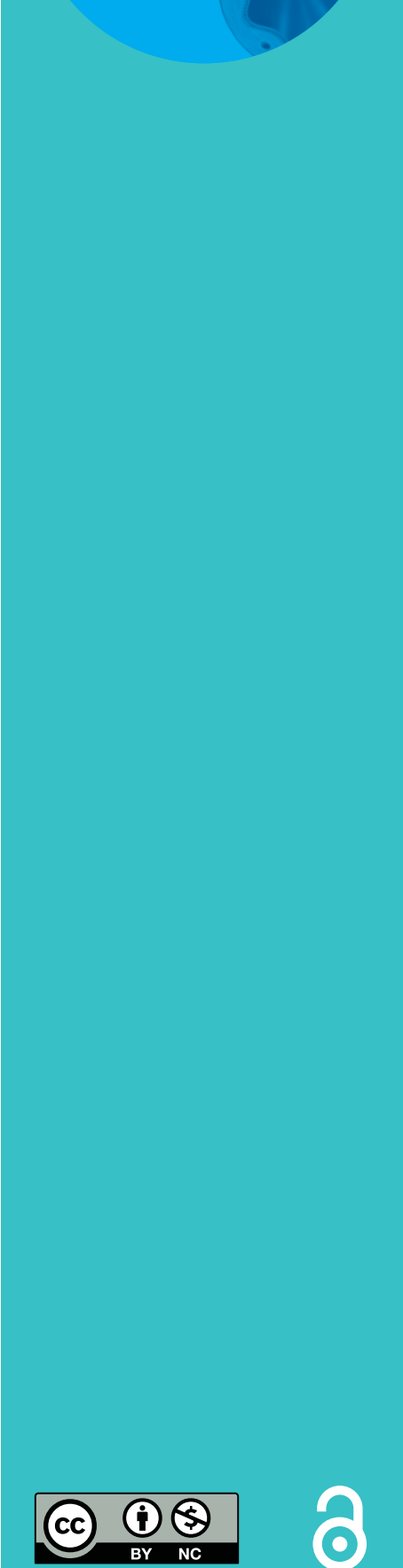

\section{Pulmonary arterial hypertension- associated changes in gut pathology and microbiota}

\author{
Ravindra K. Sharma (1) ', Aline C. Oliveira1, Tao Yang², Seungbum Kim', \\ Jasenka Zubcevic ${ }^{3}$, Victor Aquino ${ }^{1}$, Gilberto 0. Lobaton ${ }^{1}$, Ruby Goel ${ }^{1}$, \\ Elaine M. Richards ${ }^{1}$ and Mohan K. Raizada ${ }^{1}$
}

Affiliations: ${ }^{1}$ Dept of Physiology and Functional Genomics, University of Florida, Gainesville, FL, USA. ${ }^{2}$ Dept of Physiology and Pharmacology, University of Toledo, Toledo, OH, USA. ${ }^{3}$ Dept of Physiological Sciences, University of Florida, Gainesville, FL, USA.

Correspondence: Mohan K. Raizada, Dept of Physiology and Functional Genomics, University of Florida College of Medicine, PO Box 100274, Gainesville, FL 32610, USA. E-mail: mraizadaQufl.edu

ABSTRACT Emerging evidence implicates an interplay among multiple organs such as brain, vasculature, gut and lung in the development of established pulmonary arterial hypertension (PAH). This has led us to propose that activated microglia mediated-enhanced sympathetic activation contributes to PAH pathophysiology. Since enhanced sympathetic activity is observed in human PAH and the gut is highly innervated by sympathetic nerves that regulate its physiological functions, we hypothesized that PAH would be associated with gut pathophysiology.

A monocrotaline rat model of PAH was utilized to investigate the link between gut pathology and PAH. Haemodynamics, histology, immunocytochemistry and 16S RNA gene sequencing were used to assess cardiopulmonary functions, gut pathology and gut microbial communities respectively.

Monocrotaline treatment caused increased right ventricular systolic pressure, haemodynamics and pathological changes associated with $\mathrm{PAH}$. PAH animals also showed profound gut pathology that included increased intestinal permeability, increased muscularis layer, decreased villi length and goblet cells. These changes in gut pathology were associated with alterations in microbial communities, some unique to PAH animals. Furthermore, enhanced gut-neural communication involving the paraventricular nucleus of the hypothalamus and increased sympathetic drive were observed.

In conclusion, our data show the presence of gut pathology and distinct changes in gut microbiota and increased sympathetic activity in PAH. They suggest that dysfunctional gut-brain crosstalk could be critical in $\mathrm{PAH}$ and considered a future therapeutic target for $\mathrm{PAH}$.

@ERSpublications

Impaired gut-brain communication in pulmonary arterial hypertension https://bit.ly/2LpvWvY

Cite this article as: Sharma RK, Oliveira AC, Yang T, et al. Pulmonary arterial hypertensionassociated changes in gut pathology and microbiota. ERJ Open Res 2020; 6: 00253-2019 [https:// doi.org/10.1183/23120541.00253-2019].

This article has supplementary material available from openres.ersjournals.com

Received: 15 Sept 2019 | Accepted after revision: 6 May 2020

Copyright $\odot$ ERS 2020. This article is open access and distributed under the terms of the Creative Commons Attribution Non-Commercial Licence 4.0. 


\section{Introduction}

Pulmonary arterial hypertension (PAH), primarily a disease of pulmonary vasculature, is characterised by an increased pulmonary pressure that leads to right heart failure and death. PAH remains an incurable disease despite persistent efforts to develop new therapeutic targets directed towards pulmonary vasculature signalling. Our previous studies and those of others have challenged this central dogma and suggested that PAH could be a systemic disease, where coordinated interactions of multiple organ systems may be involved in the initiation and establishment of PAH pathophysiology [1,3-]. For example, our recent studies have demonstrated that microglia activation and neuroinflammation in autonomic brain regions in association with enhanced sympathetic activity play key roles in the development of PAH $[1,3]$ This led us to propose the concept of dysfunctional brain-lung communication in $\mathrm{PAH}$, consistent with evidence of increased sympathetic nerve activity (SNA) in PAH patients $[4,5]$ and involvement of neuroinflammation in many pulmonary and hypoxic pathophysiological conditions $[6,7]$.

Sympathetic nerves innervate the gastrointestinal wall and have major influences on gut motility, vasculature and microbiota thereby impacting host-microbiota cross-talk and physiological function [8]. In fact, alterations in sympathetic activity to the gut have been associated with gut microbial dysbiosis, gut pathology and overall metabolic change in many diseases, including systemic hypertension [8, 12-]. These observations, together with evidence of enhanced SNA in PAH has led us to propose that PAH is associated with alterations in gut pathology and microbiota. Therefore, the major objective of our study was to investigate the hypothesis that enhanced sympathetic activity, altered gut wall pathology and increased gut permeability, and unique alterations in gut microbial communities are associated with overall PAH pathophysiology. A minor aim was to determine if any changes in gut microbial communities were specific to PAH by comparison to systemic hypertension, a disease closely associated with alterations in the gut microbiome $[10,13,15-]$.

\section{Methods}

Animals

Eight-week-old male Sprague-Dawley (SD) rats (Charles River Laboratory) were housed in a temperature-controlled room $\left(22-25^{\circ} \mathrm{C}\right)$ with a 12:12-h light-dark cycle on autoclaved corn cob bedding. The animals were housed under specific-pathogen-free conditions and fed irradiated standard commercial rodent chow and water ad libitum. All experiments were approved by the University of Florida Institutional Animal Care and Use Committee. PAH was induced by a single subcutaneous injection of monocrotaline ((MCT) Sigma-Aldrich, St. Louis, MO, USA) at a dose of $60 \mathrm{mg}$ per $\mathrm{kg}$ of body weight, whereas rats from the control group received the equivalent volume of saline. Another set of SD rats (6-7 per group) were subcutaneously implanted with osmotic minipumps (model 2004; Durect Corporation, Cupertino, CA, USA) to infuse angiotensin II (AngII) at $200 \mathrm{ng} \cdot \mathrm{kg}^{-1} \cdot \mathrm{min}^{-1}$ (Bachem, Torrance, CA, USA) or vehicle as described previously [16]. Faecal samples were collected after 4 weeks of MCT/AngII treatment. Full details of all experimental protocols are presented in the Methods of the online "Data Supplement".

\section{Cardiopulmonary functions and autonomic evaluation}

Measurements of right ventricular systolic pressure (RVSP), $+\mathrm{dP} / \mathrm{dt},-\mathrm{dP} / \mathrm{dt}$ and right ventricular end diastolic pressure (RVEDP), tissue, blood sample collection and analysis were performed 28 days after MCT treatment as described previously [1]. We performed power spectral analysis of heart rate variability (HRV) data to determine the involvement of sympathetic activation in $\mathrm{PAH}$ using electrocardiogram recordings as described previously [1]. Two spectral components were determined: low frequency ((LF) 0.25 to $0.75 \mathrm{~Hz})$ and high frequency ((HF) 0.75 to $3.00 \mathrm{~Hz})[17,18]$. The ratio between the LF and HF was used as a representation of the balance between sympathetic and parasympathetic systems.

\section{Tyrosine hydroxylase immunostaining}

Formalin-fixed small intestine sections were incubated overnight with mouse anti-tyrosine hydroxylase (TH) antibody (AB152; 1:500; Invitrogen, Carlsbad, CA, USA) followed by incubation with Alexa Fluor 488 labelled secondary antibody (1:600; Invitrogen, Carlsbad, CA, USA).

\section{Retrograde tracing of the gut-brain neural connection}

Retrograde tracing was performed using Pseudorabies virus (PRV) to evaluate neural connections of the small intestine and the brain paraventricular nucleus (PVN) in a separate set of animals as described previously [9]. PRV tagged with green fluorescent protein (GFP); $3 \mu \mathrm{L}$ of $4.86 \times 10^{8} \mathrm{PFU} \cdot \mathrm{mL}^{-1}$ viral recombinants; $10 \mu \mathrm{L}$ ) was applied to the external surface of the small intestine and spread with a soft, sterile paintbrush. The intestine was re-positioned, abdominal muscles were sutured, and the skin was sealed with surgical wound clips. At 4 days after GFP-PRV injection, all rats were perfused, and brains were collected. Frozen $25-\mu \mathrm{m}$ sections were prepared and green fluorescence was examined in the PVN. 
ELISA

Plasma norepinephrine (NE), tissue inhibitor of metalloproteinases-1 (TIMP-1), intestinal fatty-acid binding protein (I-FABP) and high mobility group box 1 (HMGB1) were quantitated using commercially available ELISA kits. All assays were performed according to the manufacturers' instructions.

\section{Histological assessment of gut pathologies}

Histological techniques were performed to evaluate the general morphology and collagen content of small intestines as previously described [9]. Briefly, 5- $\mu \mathrm{m}$ cross-sections of jejunum were stained to quantify signs of intestinal pathologies, such as fibrosis, thickness of tunica muscularis externa, villi length and the number of goblet cells per villus.

\section{S rRNA gene sequencing of faecal samples}

Faeces were collected in sterile microfuge tubes, snap-frozen in liquid nitrogen and stored at $-80^{\circ} \mathrm{C}$ until analysis. DNA was extracted from approximately $0.25 \mathrm{~g}$ of faecal sample with a MoBio Powersoil DNA Isolation kit following the manufacturer's instructions (MoBio, Carlsbad, CA, USA). Briefly, total DNA was quantified with a Qubit 2.0 fluorometer with the dsDNA high sensitivity option, and $10 \mathrm{ng}$ of each sample was amplified by PCR using the Illumina iTag kit. PCR products were pooled and purified with the Qiagen Gel Purification Kit. Sequencing was conducted at California State University Northridge DNA sequencing facility. Reads were analysed using the QIIME 1.9.0 software package and chimeric sequences were identified using USEARCH61 [19]. Open reference operational taxonomic units (OTUs) were picked using the USEARCH61 algorithm [19], and taxonomy assignment was performed using the Greengenes $16 \mathrm{~S}$ rRNA gene database (13-5 release, 97\%) [20]. In taxonomic comparisons, OTUs unclassified at the kingdom level were discarded. All beta diversity analyses were generated from a CSS normalised OTU table within QIIME-1.9.0. A weighted UniFrac distance matrix was first generated and visualised in three-dimensional space using Emperor. ANOSIM tests for significance were calculated within QIIME-1.9.0, with the weighted UniFrac distance matrix serving as the input. Taxonomy was summarised within QIIME-1.9.0 and uploaded to the Galaxy platform for linear discriminate analysis effective size (LefSe)/cladogram enrichment plots considering significant enrichment at a $p<0.05$, Linear Discriminant Analysis (LDA) score>2.5. Closed reference OTUs were picked within QIIME-1.9.0 in order to generate functional predictions using PICRUSt [21]. Functions were summarised at level 3 and uploaded to the Galaxy platform to generate LEfSe plots where $\mathrm{p}<0.05$ and $\mathrm{LDA}>1.0$ were the criteria used for selection of enriched functions.

\section{Statistical analysis}

For all the parameters, group data were presented as mean \pm SEM and responses of RVSP, right ventricular hypertrophy (RVH) and RVEDP indices to MCT were expressed as percent changes from their control groups. Comparisons among the groups were analysed using an unpaired $t$-test and $p<0.05$ was considered as statistically significant. GraphPad Prism 8.0 (La Jolla, CA, USA) software was used to analyse the data and for graph generation.

\section{Results}

MCT-induced PAH causes gut pathology

After 4 weeks of treatment with MCT SD rats had a robust increase in RVSP $(230 \%, \mathrm{p}<0.001$, table 1$)$ and RVEDP $(80 \%, \mathrm{p}<0.01$, table 1$)$. This was associated with $\mathrm{RVH}(88 \%, \mathrm{p}<0.001$, table 1$)$. These data are

TABLE 1 Changes in haemodynamic and plasma inflammatory and permeability markers in MCT-treated rats

\begin{tabular}{lccc} 
Parameters & Control & MCT & p-value \\
\hline RVSP mmHg & $23 \pm 0.6$ & $68 \pm 5.4$ & 0.0002 \\
RVEDP $\mathrm{mmHg}$ & $2.7 \pm 0.1$ & $5.0 \pm 0.6$ & 0.002 \\
RV/LV+S g & $0.22 \pm 53$ & $525 \pm 81$ & 0.0002 \\
I-FABP $\mathrm{ng} \cdot \mathrm{mL}^{-1}$ & $229 \pm 53$ & $525 \pm 81$ & 0.012 \\
TIMP-1 $\mathrm{ng} \cdot \mathrm{mL}^{-1}$ & $10 \pm 1.0$ & $25 \pm 6.3$ & 0.004 \\
HMGB-1 $\mathrm{ng} \cdot \mathrm{mL}^{-1}$ & $119 \pm 12$ & $172 \pm 16$ & 0.029
\end{tabular}

Data are presented as mean \pm SEM ( $n=6-8$ per group), unless otherwise stated. MCT: monocrotaline; RVSP: right ventricular systolic pressure; RVEDP: right ventricular end-diastolic pressure; RV/LV+S: ratio of right ventricle to left ventricle plus septum; I-FABP: intestinal fatty acid binding protein; TIMP-1: tissue inhibitor of metalloproteinases-1; HMGB-1: high mobility group box-1 
consistent with established cardiopulmonary pathophysiology in the MCT rat model of PAH [1]. MCT-treated rats showed an eightfold increase in the ratio of LF to HF, a measure of autonomic function $(\mathrm{p}<0.001$, fig. 1a) and 2.3-fold increased plasma NE levels $(\mathrm{p}<0.01$, fig. 1b). This indicated an increase in sympathetic activity in PAH animals. Retrograde tracing with GFP-PRV was carried out to determine the neural connections between the gut and autonomic brain regions and whether they are impaired in $\mathrm{PAH}$ animals. GFP-PRV was preferentially transported to the PVN when applied to the small intestine (fig. $1 \mathrm{~d}-\mathrm{g})$. GFP labelling was very pronounced in the PVN of MCT-treated animals compared with controls. Finally, TH immunoreactivity increased $\sim 2.8$-fold $(\mathrm{p}<0.001$, fig. $1 \mathrm{c}, \mathrm{h}$ and $\mathrm{i})$ in the intestine of MCT-treated animals.

Next, we determined whether increased sympathetic activity in PAH animals was associated with gut permeability and pathology. Intestinal fatty acid binding protein (I-FABP), an epithelial protein, is released into circulation as a result of mucosal damage and is used as marker for gut permeability [22]. We observed a 2.3-fold elevation of plasma I-FABP in MCT-treated animals $(p<0.05$, table 1$)$. We also measured plasma tissue inhibitor of metalloproteinases-1 (TIMP-1) and high mobility group box-1 (HMGB1), two proteins increased in PAH [23, 24] to verify our MCT model. As expected, TIMP-1 increased 2.6-fold $(\mathrm{p}<0.001)$ and HMGB1 increased 1.4 -fold $(\mathrm{p}<0.05)$ in MCT-treated compared to control animals.
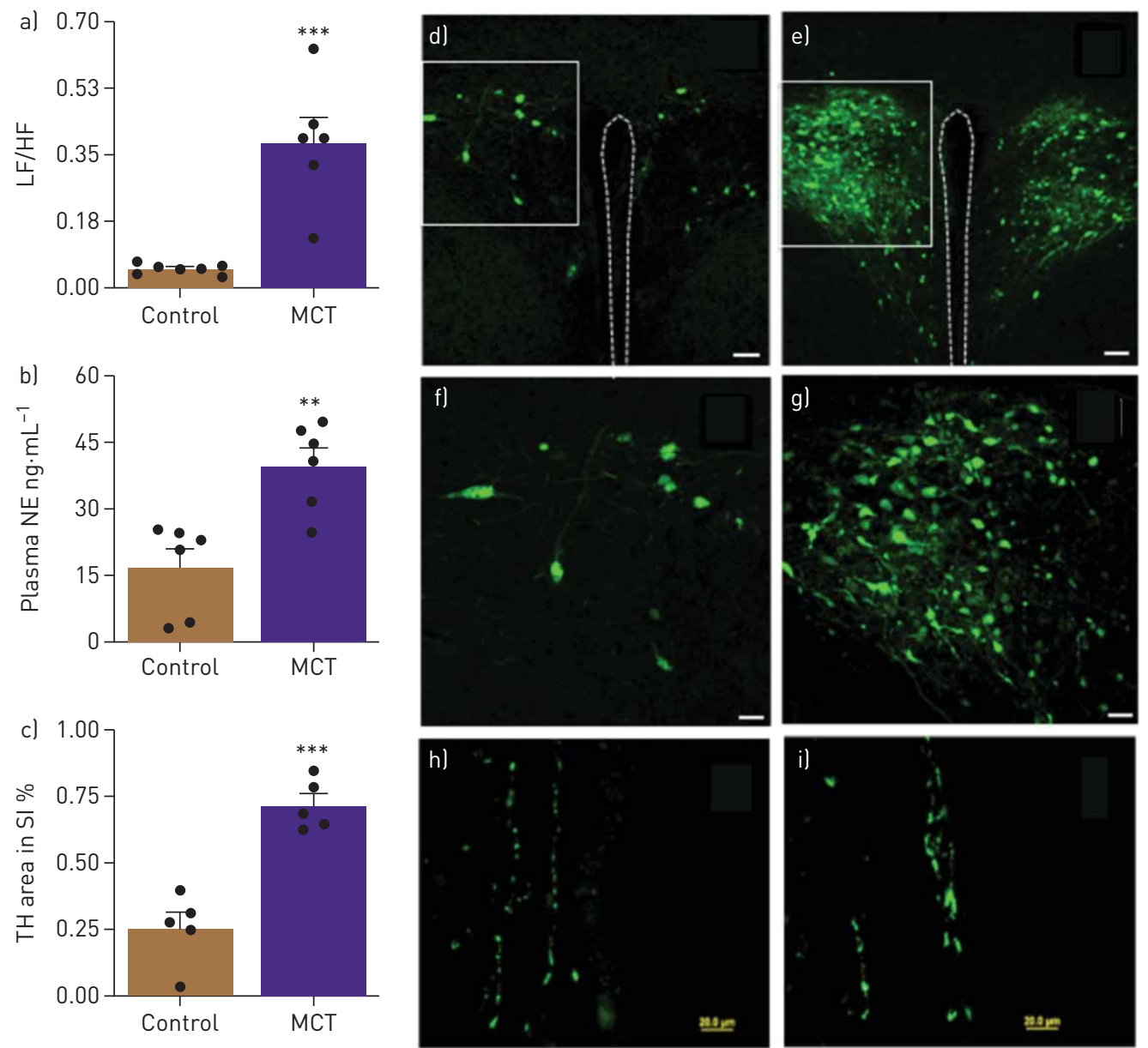

FIGURE 1 a) Ratio of low frequency ((LF) reflecting sympathetic activity) and high frequency ((HF) reflecting parasympathetic activity) heart rate variability and (b) Plasma norepinephrine (NE) shows autonomic imbalance in monocrotaline (MCT)-treated rats. c) Cumulative tyrosine hydroxylase (TH)-positive staining, an index of catecholamine synthesis, was significantly increased in small intestines of MCT-challenged rats. d,e) Brain-gut neural connections are enhanced in MCT-induced pulmonary arterial hypertension (PAH). A neural circuit between the gut and the brain paraventricular nucleus (PVN) was identified by retrograde tracing using green fluorescent protein (GFP) Pseudorabies virus (GFP-PRV). Representative micrograph showing GFP expression in the PVN of control and MCT rats (scale bar, $50 \mu \mathrm{m}$ ). f,g) High-resolution micrographs show increased GFP in PVN of MCT rats compared with controls (scale bar: $150 \mu \mathrm{m}$ ). $h$,i) Representative micrographs showing TH immunoreactivity in the small intestine (scale bar, $20 \mu \mathrm{M}$ ). ${ }^{* * *}$ : $p<0.001, * *: p<0.01$ versus control ( $n=5-7$ rats per group). Data are represented as mean \pm SEM. 
Finally, we examined the small intestine in MCT-treated animals for pathology. PAH animals showed significantly increased intestinal fibrosis $(143 \%, \mathrm{p}<0,001$, figure $2 \mathrm{a}, \mathrm{b}$ and $\mathrm{c}$ ) and muscularis thickness $(37 \%, \mathrm{p}<0.001$, figure $2 \mathrm{~d}$, e and f). Moreover, decreases in the number of goblet cells $(15 \%, \mathrm{p}<0.05$, figure $2 \mathrm{~g}, \mathrm{~h}$, and i) and villus length $26 \%, \mathrm{p}<0.001$, figure $2 \mathrm{j}, \mathrm{k}$ and $\mathrm{l}$ ) were observed in PAH animals.

\section{Gut microbial communities are altered in PAH animals}

Gut pathology is a major influence on gut microbiota with significant outcomes on host-microbiota cross-talk and overall gut homeostasis. This, coupled with previous evidence of the involvement of microbiota in various pulmonary diseases, led us to compare gut microbiota of PAH and control SD rats. Three-dimensional principle coordinate analysis (PCoA) showed significant separation of microbiota of MCT-treated animals from that of controls $(\mathrm{p}=0.031$, figure $3 \mathrm{a})$. The ratio of Firmicutes to Bacteroidetes (F/B) was increased in MCT-treated rats compared with controls $(\mathrm{p}<0.05$, figure $3 \mathrm{~b})$. A cladogram and LEfSe demonstrated interesting differences between the groups (figure $3 \mathrm{c}$ and $\mathrm{d}$ ), some of which are summarised as follows: control rats demonstrated predominance of Enterobacteriaceae, a large family of Gram-negative, benign, beneficial symbiotic bacteria. In contrast, numerous microbial genera were observed in MCT-treated rats that are known to contain pathogenic species including a few associated with pulmonary diseases [25, 26]. Among them are Gram-positive, aerobic, Corynebacteriaceae,
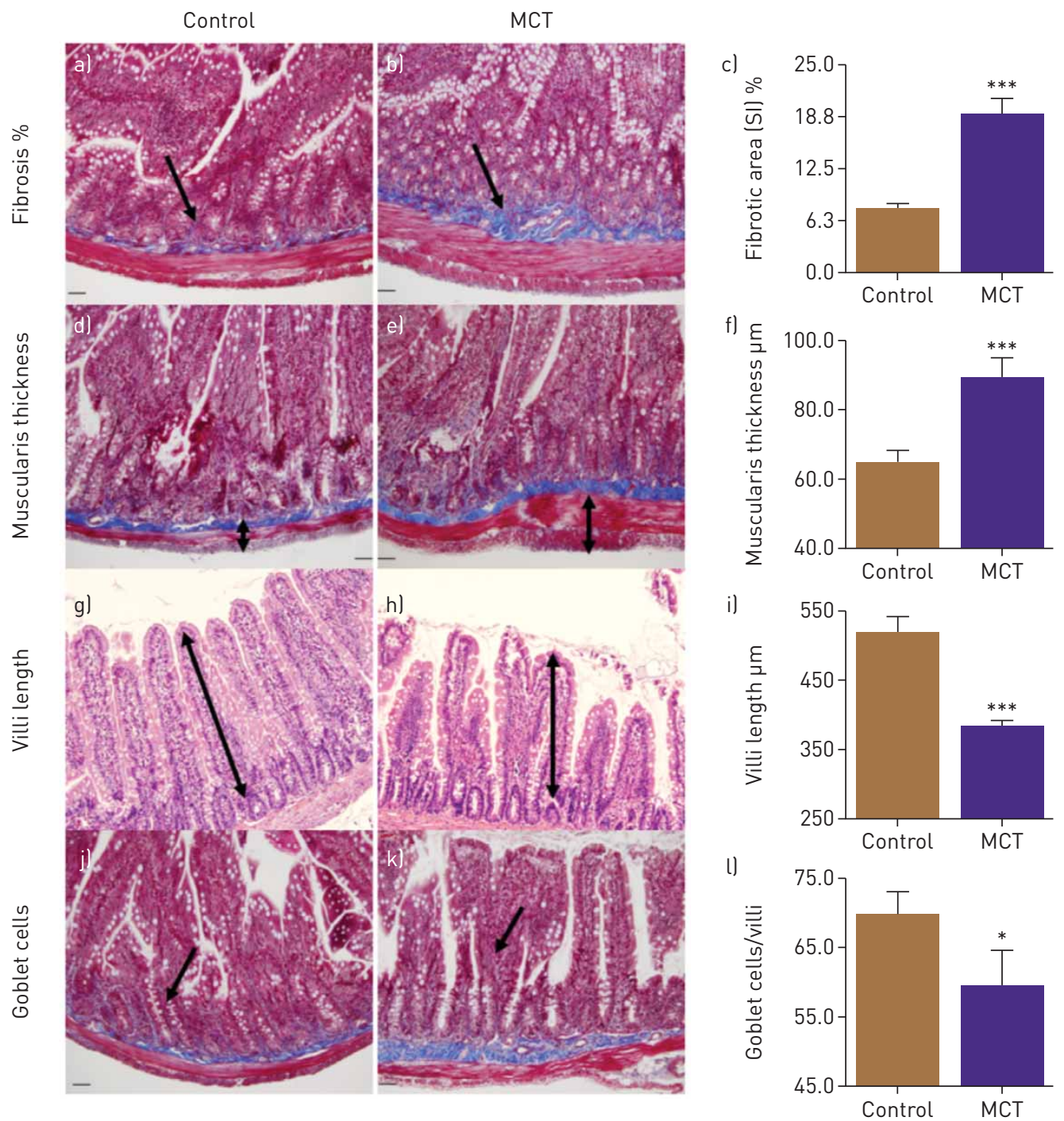

FIGURE 2 Gut pathologies were determined histologically. a-c) Masson's trichrome stain showed increased fibrosis in small intestine (SI) of monocrotaline (MCT) rats. d-f) Haematoxylin and eosin staining revealed increased muscularis layer thickness of $\mathrm{SI}$ in these rats, whereas villi length ( $\mathrm{g}$-i) and number of goblet cells $(j-l)$ were decreased in MCT rats compared to control. ${ }^{* * *}: p<0.001,{ }^{*}: p<0.05$ versus control ( $n=6$ rats per group). Data are represented as meanıSEM. 
a) PC2 (16.2\%)

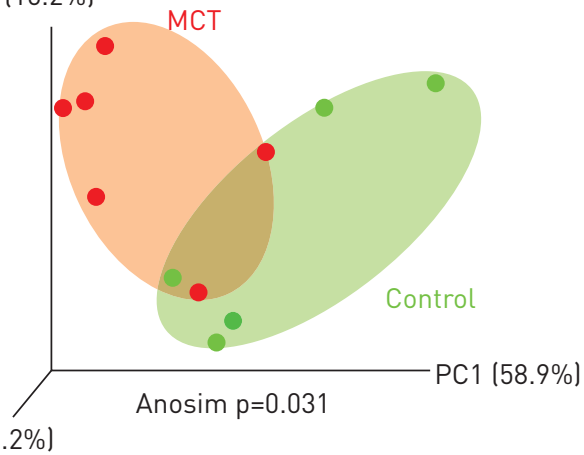

PC3 (7.2\%)

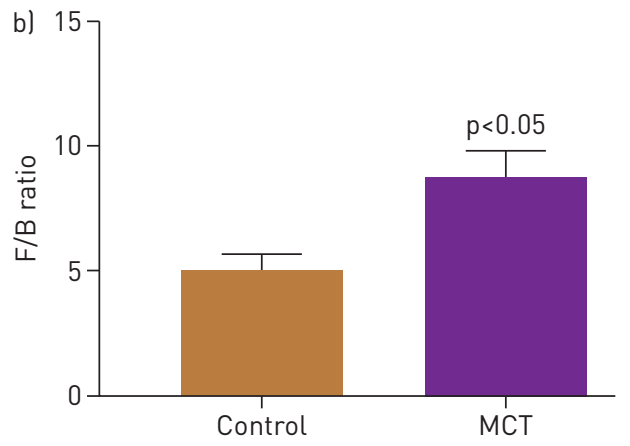

c)

$$
\begin{aligned}
& \text { Control } \\
& \text { MCT }
\end{aligned}
$$

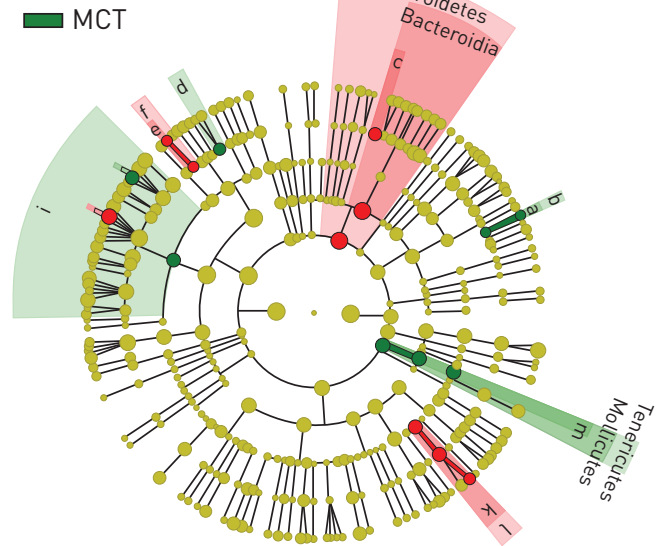

d)

$$
\begin{aligned}
& \square \text { a: Corynebacterium } \\
& \square \text { b: Corynebacteriaceae } \\
& \square \text { c: S24_7 } \\
& \square \text { d: Aerococcaceae } \\
& \square \text { e: Streptococcus } \\
& \square \text { f: Streptococcaceae } \\
& \square \text { g: Clostridium } \\
& \square \text { h: Blautia } \\
& \square \text { i: Clostridiales } \\
& \square \text { j: Proteus } \\
& \square \text { k: Enterobacteriaceae } \\
& \square \text { l: Enterobacteriales } \\
& \square \text { m: RF39 }
\end{aligned}
$$

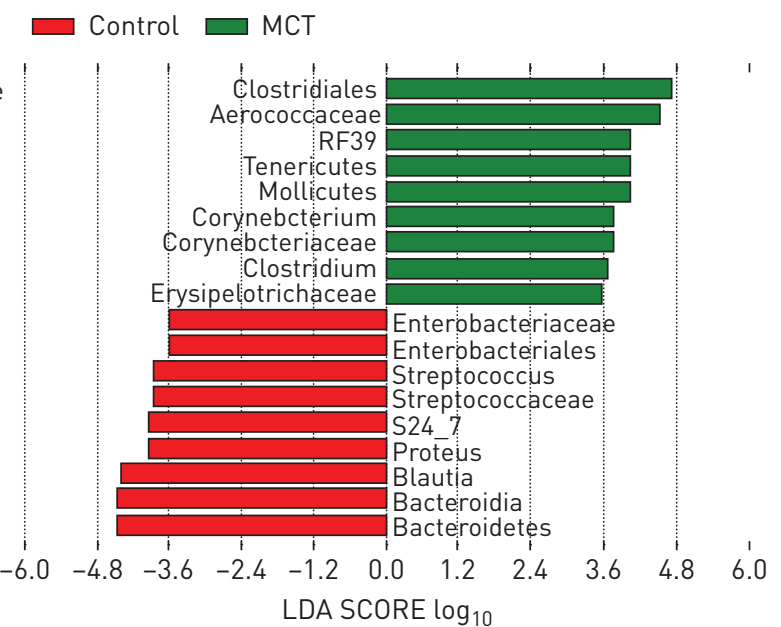

FIGURE 3 Effects of monocrotaline (MCT)-induced PAH on microbial communities. Bacterial 16S rRNA genes were analysed from faecal samples to characterise gut microbiomes. a) Three-dimensional principal coordinate analysis (PCoA) showed that control and MCT-treated cohorts have significantly distinct bacterial populations. b) Firmicutes (F) to Bacteroidetes (B) ratio (F/B) was increased in MCT rats, indicating gut dysbiosis. c) A cladogram showing family- and genus-level changes of bacteria in each group. d) Linear discriminant analysis effect size (LEfSe) was used to determine the bacteria most likely to explain differences between cohorts. *: $p<0.05$ versus control ( $n=5-6$ rats per group).

Corynebacterium. The class Erysipelotrichaceae, in the Firmicutes phylum, which includes the Clostridium, Turicibacter, and Mollicutes genera. Erysipelotrichaceae are involved in gut dysbiosis in diseases such as inflammatory bowel disease (IBD), obesity, and metabolic disease [27, 29-].

\section{Distinct microbial communities in MCT-treated versus chronic angiotensin II-treated SD rats}

We compared changes in gut bacterial communities in SD rats with PAH (MCT treatment) and with systemic hypertension (chronic AngII infusion). First, we performed network analysis of genera in PAH and hypertensive animals in comparison to their respective normotensive controls. We observed a cluster of bacterial genera enriched in the MCT-treated group (blue circle) that was distinct from control animals (red circles, figure 4a). Similarly, hypertensive animals showed distinct clustering from their controls (figure $4 \mathrm{~g}$ ). Next, we compared relative levels of bacterial genera and observed differences between the hypertensive and PAH animals. For example, we observed profoundly increased Bifidobacterium and Streptococcus in hypertensive animals, while Oscillospira, Roseburia and Akkermansia were increased in $\mathrm{PAH}$ animals (figure $4 \mathrm{c}, \mathrm{d}$ and f). These data indicate several genera unique to each disease.

\section{Discussion}

This study provided three novel observations: 1) Enhanced sympathetic nervous system activity and gut to brain (PVN) connections in PAH animals; 2) Altered gut pathology, microbial communities and increased gut permeability in PAH animals; and 3) significant differences in disease-associated bacterial genera between PAH animals and animals with systemic hypertension.

Evidence for impaired sympathetic gut communication includes: 1) GFP-PRV applied to the small intestine was rapidly retrogradely transported to the neurons of the PVN to a greater degree in PAH animals than in control 

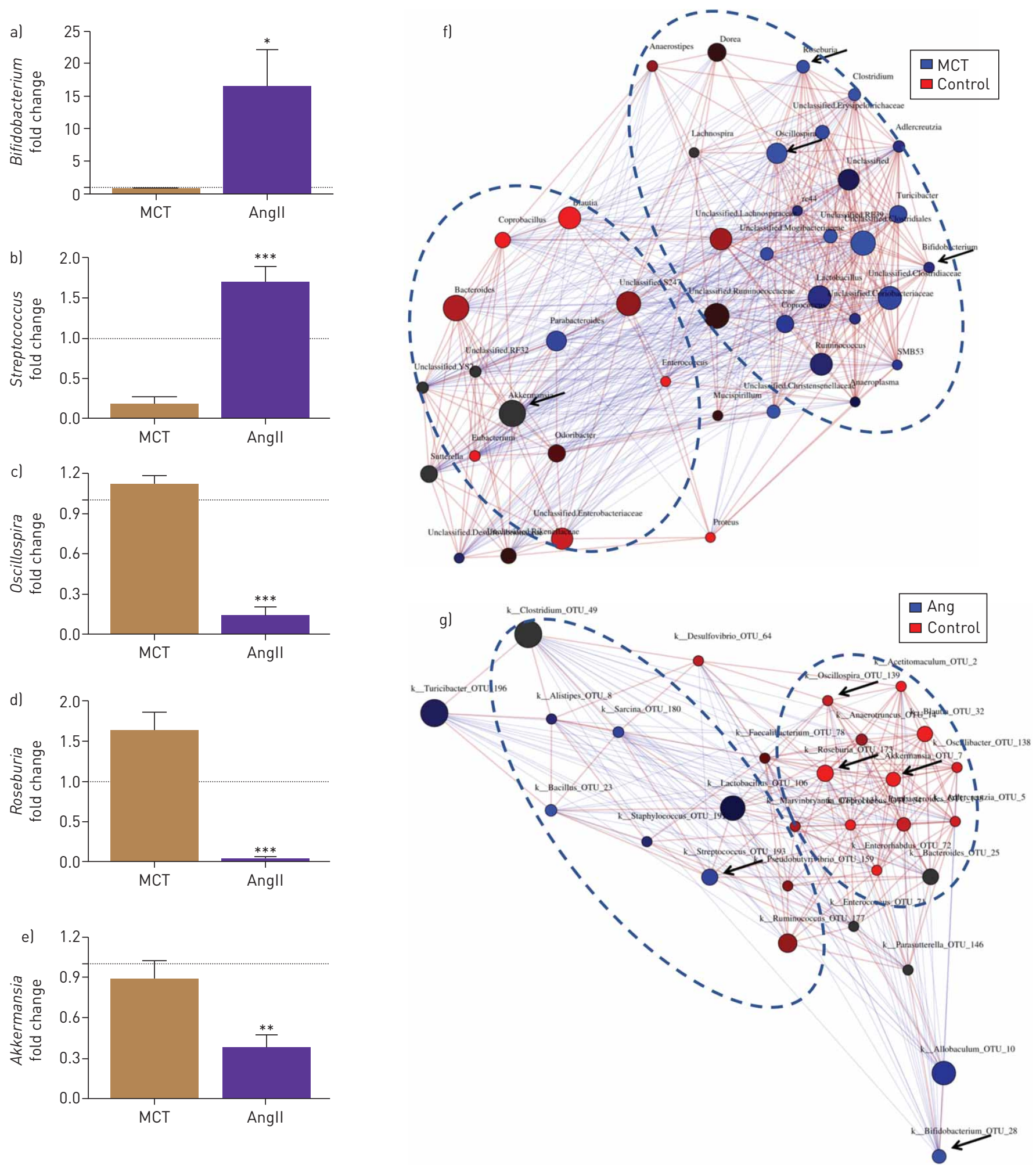

FIGURE 4 Comparison of the microbial community in monocrotaline (MCT)-induced pulmonary arterial hypertension (PAH) rats and angiotensin II (Angll)-induced hypertensive rats. Network analysis and interaction of bacterial abundance showed that the bacterial groups enriched in Angll-induced hypertensive rats were: (a) Bifidobacterium and (b) Streptococcus, in contrast to MCT-PAH rats, whereas, the bacterial group enriched in MCT-PAH rats were: (c) Oscillospira, (d) Roseburia and (e) Akkermansia when compared to Angll-HTN rats. Fold changes were determined by dividing the bacterial percentage in each MCT or Angll-treated individual rat by average bacterial percentage in MCT or Angll controls, respectively. The dotted lines indicate a fold change value of 1 , the average value of control group. MCT or Angll groups have higher (>1) or lower $(<1)$ levels of indicated bacterial genus than in control group. ${ }^{* * *}: p<0.001,{ }^{* *}: p<0.01,{ }^{*}: p<0.05$ versus MCT ( $n=6$ rats per group). 
animals. This may reflect alterations in neuronal activity, cellular metabolism, or polysynaptic transmission in $\mathrm{PAH}$, as all are known modulators of PRV transport. Involvement of activated microglia and neuroinflammation, known to alter neuronal activity, in the development of PAH pathophysiology is consistent with this view [1]. 2) Increased LF/HF ratio, reflecting greater sympathetic tone, together with higher plasma NE suggests raised sympathetic drive in these animals as we reported previously [1]. This finding is also consistent with increased SNA observed in PAH patients that independently predicts severity of disease [30]. 3) Increased TH in the intestines of PAH animals, together with enhanced sympathetic innervation of the gut in $\mathrm{PAH}$ animals, support our contention of an impaired gut-brain-lung axis in the pathophysiology of PAH.

Our data, for the first time, demonstrate that MCT-induced PAH is associated with profound gut wall pathology. This includes increased fibrosis and muscular tissue in the gut wall, stunted villi and decreased goblet cells. These changes have significant implications for host-microbiome interactions that potentially influence brain-gut-lung homeostasis. For example, intestinal villi increase surface area for absorption of nutrients in addition to harbouring digestive enzymes. Thus, their shortening in PAH animals is likely to compromise metabolism and nutrient absorption. Furthermore, such impaired absorption may provide an altered milieu allowing adverse microbial communities to bloom (discussed below). Goblet cells produce mucin that principally protects the gut from pathogenic microbiota and thus regulates the immune response of the gut [31]. Thus, decreases in goblet cells in PAH animals may decrease mucin production, contracting mucin-degrading bacterial communities and expanding harmful ones. There are many examples of flawed mucin and gut microbiota relationships in diseased states to support this concept [32, 33]. However, further experiments are warranted to confirm this in PAH. Nonetheless, it is evident from our and others' data that gut pathology increased gut permeability $[9,34,35]$.

Our data are in general agreement with an earlier study of gut microbiota in a different rat model of PAH [36]. There was little difference in alpha diversity between the control and PAH animals, whereas the F/B ratio was significantly increased. A significantly different clustering of microbial genera from control and MCT-treated PAH animals was observed. Importantly, Bacteroidetes were depleted in MCT rats consistently with the previous observation [36], resulting in increased F/B ratios in both PAH models. Bacteroidetes, Gram-negative bacteria, enriched in healthy rats, are indicative of a balanced gut. Depletion of this phylum may have major mechanistic implications in $\mathrm{PAH}$ and repopulating them by probiotic-based delivery may be therapeutic via the brain-gut-lung axis. Furthermore, PAH rats had increased Clostridiales and Aerococcaceae bacteria of the Firmicutes phylum. Clostridiales, a potential respiratory pathogen, was reported to be enriched in individuals exposed to agricultural dust high in endotoxin and muramic acid [37]. Similarly, Aerococcaceae were enriched in Crohn's disease patients [38]. These microbial communities were not prominent in gut microbiota of control animals.

Gut microbial dysbiosis and changes in gut permeability have recently been linked to multiple diseases and pathophysiological conditions including cardiopulmonary disease, hypertension, obesity, diabetes, and metabolic disorders, implicating a role for altered gut microbiota in pathogenesis of diverse diseases $[9,15$, 36, 39-42]. However, there is a paucity of data identifying disease-specific changes in gut microbial communities. Our study addresses this issue by comparing two related diseases, PAH and systemic hypertension. In addition to PAH- and systemic hypertension-specific clustering of microbial communities at the genus level, we demonstrate significant differences between the two pathological states. First, alpha diversity in hypertensive animals was significantly decreased [15, 43]. No significant change in this parameter was observed in PAH animals, an observation consistent with a previous study [36]. Second, hypertensive animals showed significant depletion of bacterial communities of the genera Roseburia, Oscillospira and Akkermansia while PAH animals showed depletion of Bifidobacterium and Streptococcus. In contrast, Oscillospira and Roseburia are enriched in PAH. These genera are associated with short chain fatty acid production and lean body mass and are generally considered to be beneficial bacterial communities [44, 45]. The relevance of this observation needs further investigation. However, it is reasonable to postulate that changes in one or more bacterial genera may precipitate a cascade of intricate microbiota changes altering overall host-microbiota cross-talk in PAH. Therefore, metagenomic analysis and expansion of knowledge about functional aspects of microbial communities would provide critical information in this regard as pointed out recently [46]. We used the well accepted MCT rat model of PAH in this study; it represents a complex multiorgan disease process that culminates in PAH and associated right heart failure. MCT has been used in PAH research for over 40 years even though its mechanism of action remains poorly understood. We acknowledge that the effect of MCT observed in this study may not be direct. However, this is unlikely as a result of other supporting observations. 1) Gut dysbiosis has been observed in a Sugen-hypoxia rat model of PAH [36]. 2) We demonstrated the presence of gut dysbiosis and leakiness in patients with $\mathrm{PAH}$ [37]. 3) Hypoxia-induced $\mathrm{PAH}$ in mice is associated with neuroinflammation, increased sympathetic activity, gut microbial dysbiosis and gut pathology [47]. Therefore, this concept awaits further validation by utilisation of other animal models of PAH. 
In summary, these observations indicate that sympathetic overactivation, gut leakiness, pathology and specific changes in gut microbial communities are associated with MCT-induced PAH pathophysiology. It also provides evidence of differences in microbial communities between hypertensive and PAH animals. Obviously, these observations need to be confirmed in another animal model of PAH and with metagenomic studies to validate this concept of dysfunctional brain-gut-lung communication in PAH.

\section{Conclusions}

Accumulating evidence suggest that PAH could be considered a systemic disease rather than a disease of the pulmonary vasculatures and a coordinated interaction among multiple organs may be critical in the development of this disease. Our study was designed to test the hypothesis that altered communication among the autonomic brain region-gut and lungs is important in the development of PAH. Evidence show that PAH is associated with increased gut-hypothalamic paraventricular nucleus trafficking, altered gut wall pathology and imbalanced gut microbiota. This provides support for an impaired gut-brain-lung cross-talk in PAH. This evidence open a new avenue for therapeutic interventions for PAH therapy.

Conflict of interest: R.K. Sharma has nothing to disclose. A.C. Oliveira has nothing to disclose. T. Yang has nothing to disclose. S. Kim has nothing to disclose. J. Zubcevic has nothing to disclose. V. Aquino has nothing to disclose G.O. Lobaton has nothing to disclose. R. Goel has nothing to disclose. E.M. Richards has nothing to disclose M.K. Raizada reports grants from the NIH outside the submitted work.

Support statement: This study was supported by National Heart, Lung, and Blood Institute grant HL102033. Funding information for this article has been deposited with the Crossref Funder Registry.

\section{References}

1 Sharma RK, Oliveira AC, Kim S, et al. Involvement of neuroinflammation in the pathogenesis of monocrotaline-induced pulmonary hypertension. Hypertension 2018; 71: 1156-1163.

2 Gurtu V, Michelakis ED. A paradigm shift is needed in the field of pulmonary arterial hypertension for its entrance into the precision medicine era. Circ Res 2016; 119: 1276-1279.

3 Oliveira AC, Sharma RK, Aquino V, et al. Involvement of microglial cells in hypoxia-induced pulmonary hypertension. Am J Respir Cell Mol Biol 2018; 59: 271-273.

$4 \quad$ Kishi T. Heart failure as an autonomic nervous system dysfunction. J Cardiol 2012; 59: 117-122.

5 Velez-Roa S, Ciarka A, Najem B, et al. Increased sympathetic nerve activity in pulmonary artery hypertension. Circulation 2004; 110: 1308-1312.

6 Johnson SM, Randhawa KS, Epstein JJ, et al. Gestational intermittent hypoxia increases susceptibility to neuroinflammation and alters respiratory motor control in neonatal rats. Respir Physiol Neurobiol 2018; 256 $128-142$.

7 Wang $\mathrm{H}$, Blackall $\mathrm{M}$, Sominsky $\mathrm{L}$, et al. Increased hypothalamic microglial activation after viral-induced pneumococcal lung infection is associated with excess serum amyloid A production. J Neuroinflammation 2018; 15: 200.

8 Zubcevic J, Richards EM, Yang T, et al. Impaired autonomic nervous system-microbiome circuit in hypertension. Circ Res 2019; 125: 104-116.

9 Santisteban MM, Qi Y, Zubcevic J, et al. Hypertension-linked pathophysiological alterations in the gut. Circ Res 2017; 120: 312-323.

10 Toral M, Robles-Vera I, de la Visitación N, et al. Critical role of the interaction gut microbiota - sympathetic nervous system in the regulation of blood pressure. Front Physiol 2019; 10: 231.

11 Straub RH, Wiest R, Strauch UG, et al. The role of the sympathetic nervous system in intestinal inflammation. Gut 2006; 55: 1640-1649.

12 Cervi AL, Lukewich MK, Lomax AE. Neural regulation of gastrointestinal inflammation: role of the sympathetic nervous system. Auton Neurosci 2014; 182: 83-88.

13 Toral M, Robles-Vera I, de la Visitación N, et al. Role of the immune system in vascular function and blood pressure control induced by faecal microbiota transplantation in rats. Acta Physiol (Oxf) 2019; 227: e13285.

14 Kim S, Goel R, Kumar A, et al. Imbalance of gut microbiome and intestinal epithelial barrier dysfunction in patients with high blood pressure. Clin Sci (Lond) 2018; 132: 701-718.

15 Yang T, Santisteban MM, Rodriguez V, et al. Gut dysbiosis is linked to hypertension. Hypertension 2015; 65: $1331-1340$.

16 Sharma RK, Yang T, Oliveira AC, et al. Microglial cells impact gut microbiota and gut pathology in angiotensin ii-induced hypertension. Circ Res 2019; 124: 727-736.

17 Murasato Y, Hirakawa H, Harada Y, et al. Effects of systemic hypoxia on R-R interval and blood pressure variabilities in conscious rats. Am J Physiol 1998; 275: H797-H804.

18 Waki H, Katahira K, Polson JW, et al. Automation of analysis of cardiovascular autonomic function from chronic measurements of arterial pressure in conscious rats. Exp Physiol 2006; 91: 201-213.

19 Edgar RC. Search and clustering orders of magnitude faster than BLAST. Bioinformatics 2010; 26: 2460-2461.

20 DeSantis TZ, Hugenholtz P, Larsen N, et al. Greengenes, a chimera-checked 16S rRNA gene database and workbench compatible with ARB. Appl Environ Microbiol 2006; 72: 5069-5072.

21 Langille MG, Zaneveld J, Caporaso JG, et al. Predictive functional profiling of microbial communities using 16S rRNA marker gene sequences. Nat Biotechnol 2013; 31: 814-821.

22 Lau E, Marques C, Pestana D, et al. The role of I-FABP as a biomarker of intestinal barrier dysfunction driven by gut microbiota changes in obesity. Nutr Metab (Lond) 2016; 13: 31. 
23 Lepetit H, Eddahibi S, Fadel E, et al. Smooth muscle cell matrix metalloproteinases in idiopathic pulmonary arterial hypertension. Eur Respir J 2005; 25: 834-842.

24 Sadamura-Takenaka Y, Ito T, Noma S, et al. HMGB1 promotes the development of pulmonary arterial hypertension in rats. PLoS One 2014; 9: e102482.

25 Huang YJ, Lynch SV. The emerging relationship between the airway microbiota and chronic respiratory disease: clinical implications. Expert Rev Respir Med 2011; 5: 809-821.

26 Fujimura KE, Lynch SV. Microbiota in allergy and asthma and the emerging relationship with the gut microbiome. Cell Host Microbe 2015; 17: 592-602.

27 Matsuoka K, Kanai T. The gut microbiota and inflammatory bowel disease. Semin Immunopathol 2015; 37: 47-55.

28 Guo X, Li J, Tang R, et al. High fat diet alters gut microbiota and the expression of Paneth cell-antimicrobial peptides preceding changes of circulating inflammatory cytokines. Mediators Inflamm 2017; 2017: 9474896.

29 Clarke SF, Murphy EF, Nilaweera K, et al. The gut microbiota and its relationship to diet and obesity: new insights. Gut Microbes 2012; 3: 186-202.

30 Ciarka A, Doan V, Velez-Roa S, et al. Prognostic significance of sympathetic nervous system activation in pulmonary arterial hypertension. Am J Respir Crit Care Med 2010; 181: 1269-1275.

31 Pelaseyed T, Bergström JH, Gustafsson JK, et al. The mucus and mucins of the goblet cells and enterocytes provide the first defense line of the gastrointestinal tract and interact with the immune system. Immunol Rev 2014 260: 8-20.

32 Corfield AP. The interaction of the gut microbiota with the mucus barrier in health and disease in human. Microorganisms 2018; 6: E78.

33 Schroeder BO. Fight them or feed them: how the intestinal mucus layer manages the gut microbiota. Gastroenterol Rep (Oxf) 2019; 7: 3-12.

34 Lee SH. Intestinal permeability regulation by tight junction: implication on inflammatory bowel diseases. Intest Res 2015; 13: 11-18.

35 Lee B, Moon KM, Kim CY. Tight junction in the intestinal epithelium: its association with diseases and regulation by phytochemicals. J Immunol Res 2018; 2018: 2645465.

36 Callejo M, Mondejar-Parreño G, Barreira B, et al. Pulmonary arterial hypertension affects the rat gut microbiome. Sci Rep 2018; 8: 9681.

37 Goel R, Kim S, Rigatto K, et al. Increased gut dysbiosis and leakiness in patients with pulmonary arterial hypertension. Circulation 2017; 136: A20620.

38 Kiley JP, Caler EV. The lung microbiome. A new frontier in pulmonary medicine. Ann Am Thorac Soc 2014; 11 Suppl 1: S66-S70.

39 Ranchoux B, Bigorgne A, Hautefort A, et al. Gut-lung connection in pulmonary arterial hypertension. Am J Respir Cell Mol Biol 2017; 56: 402-405.

40 Nagpal R, Newman TM, Wang S, et al. Obesity-linked gut microbiome dysbiosis associated with derangements in gut permeability and intestinal cellular homeostasis independent of diet. J Diabetes Res 2018; 2018: 3462092.

41 Martinez KB, Leone V, Chang EB. Western diets, gut dysbiosis, and metabolic diseases: are they linked? Gut Microbes 2017; 8: 130-142.

42 Thenappan T, Khoruts A, Chen Y, et al. Can intestinal microbiota and circulating microbial products contribute to pulmonary arterial hypertension? Am J Physiol Heart Circ Physiol 2019; 317: H1093-H1101.

43 Li J, Zhao F, Wang Y, et al. Gut microbiota dysbiosis contributes to the development of hypertension. Microbiome 2017; 5: 14.

44 de la Cuesta-Zuluaga J, Mueller NT, Álvarez-Quintero R, et al. Higher fecal short-chain fatty acid levels are associated with gut microbiome dysbiosis, obesity, hypertension and cardiometabolic disease risk factors. Nutrients 2018; 11: E51.

45 Konikoff T, Gophna U. Oscillospira: a central, enigmatic component of the human gut microbiota. Trends Microbiol 2016; 24: 523-524.

46 Jama H, Kaye DM, Marques FZ. Population-based gut microbiome associations with hypertension. Circ Res 2018 123: $1185-1187$.

47 Sharma RK, Oliveira AC, Yang T. Gut pathology and its rescue by ACE2 (Angiotensin-Converting Enzyme 2) in hypoxia-induced pulmonary hypertension. Hypertension 2020; 76: 206-216. 